\title{
Erratum to: Melatonin Alleviates Acute Gouty Inflammation In Vivo and In Vitro"
}

\author{
Wen-ze XIAO ${ }^{1,2}$, Li ZHAO ${ }^{1}$, Lin $\mathrm{CAO}^{1}$, Xiao-xia ZHU ${ }^{1}$, He-jian ZOU ${ }^{1 \#}$ \\ ${ }^{I}$ Department of Rheumatology and Immunology, Huashan Hospital Affiliated to Fudan University, Shanghai 200040, China \\ ${ }^{2}$ Department of Rheumatology and Immunology, Fudan University Pudong Medical Center, Shanghai 201300, China
}

(C) Huazhong University of Science and Technology 2023

\section{Current Medical Science}

https://link.springer.com/article/s11596-021-2362-x

Published in Aug. 2021

The authors examined the original data of their work and noticed some mistakes. The ELISA kit was bought from IBL Co. Germany instead of Abcam Co, UK. The anti-NLRP3, anti-IL-1 $\beta$ and anti-caspase-1 primary antibodies were from CST Co, USA. There were 31 acute gout patients and 25 gout patients in remission who met the American Rheumatism Society (ACR) diagnostic criteria of gout in this study, and 7 normal individuals were recruited as controls. The authors sincerely apologize for the mistakes and confirm that the changes do not affect the scientific conclusion of the published work. 